\title{
ABSOLUTE CONTINUITY, SINGULARITY AND PRODUCT MEASURES
}

\author{
ROY A. JOHNSON \\ Department of Mathematics \\ Washington State University \\ Pullman, Washington 99164 \\ U.S.A.
}

(Received May 25, 1982)

\begin{abstract}
Conditions are given under which a product of two semifinite measures is absolutely continuous or weakly singular with respect to another product of two semifinite measures. A Lebesgue type decomposition theorem is proved for certain product measures so that the resulting measures are themselves product measures.

KEY WORDS AND PHRASES. Absolutely continuous, weakly singular, smallest product, largest product, quasi-dominant, strongly recessive.

1980 MATHEMATICS SUBJECT CLASSIFICATION CODES. Primary 28 A 35.
\end{abstract}

\section{INTRODUCTION}

The main results of this paper are Theorems 4.2 and 4.3 in which we observe conditions under which the Lebesgue decomposition of one product measure with respect to another yields measures which are themselves product measures. It will be seen that the expected results (similar to those of [1]) hold for the smallest product of two semifinite measures but that some surprising things can happen for the largest product of two semifinite measures.

Throughout this paper $\mu$ and $\mu^{\prime}$ will be (nonnegative, countably additive) measures on a sigma-ring $\delta$, and $\nu$ and $\nu^{\prime}$ will be measures on a sigma ring $J$. We say that $\mu$ is absolutely continuous with respect to $\mu^{\prime}$, denoted $\mu^{\prime} \ll \mu^{\prime}$, if $\mu(E)=0$ whenever $E \in S$ and $\mu^{\prime}(E)=0$. We say that $\mu$ is weakly singular with respect to $\mu^{\prime}$, denoted $\mu S \mu^{\prime}$, if for each $E \in S$, there exists $F \in S$ such that $\mu(E)=\mu(E \cap F)$ and $\mu^{\prime}(F)=0$. A measure is semifinite if every set of infinite measure contains sets of arbitrarily large positive measure. Undefined terminology and notation can be found in [2]. 
A product of $\mu$ and $\nu$ is a measure $(\mu \times \nu)^{\#}$ on $\delta \times J$ such that $(\mu \times \nu)^{\#}=$ $\mu(G) \nu(H)$ whenever $G \in S, H \in J$ and $\mu(G) \nu(H)<\infty$. (Such a product is called a pseudo-product measure in [3].) We avoid requiring that $(\mu \times \nu)^{\#}(G \times H)=$ $\mu(G) \nu(H)$ for all $G$ in $S$ and $H$ in $J$ on the grounds that (i) the important sets for a measure are usually the sets of finite measure, (ii) if $(\mu \times \nu){ }^{\#}$ and

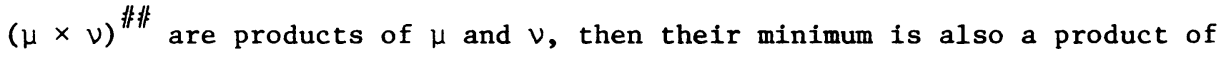
$\mu$ and $\nu$ under this definition, and (iii) such theorems as Theorem 4.2 and 4.3 hold if we use this less restrictive definition of product measure. Of course, if $\mu$ and $\nu$ are both semifinite, then we automatically get the stronger property that $(\mu \times \nu)^{\#}(G \times H)=\mu(G) \nu(H)$ for all $G$ in $S$ and $H$ in $J$.

The smallest product of $\mu$ and $\nu$ is the unique measure $(\mu \times \nu)$ on $g \times J$ such that

(i) $(\mu \times \nu)^{S}(G \times H)=\mu(G) \nu(H)$ whenever $\mu(G)<\infty$ and $\nu(H)<\infty$ and (ii) $(\mu \times \nu)^{S}(M)=\sup \left\{(\mu \times \nu)^{S}(M \cap(G \times H)): \mu(G)<\infty\right.$ and $\left.\nu(H)<\infty\right\}$ [4, Theorem 39.1]. The largest product of $\mu$ and $\nu$ is the measure $(\mu \times \nu)^{L}$ on $S \times J$ defined by the formula

$$
(\mu \times \nu)^{L}(M)=\inf \left\{\Sigma_{1}^{\infty} \mu\left(G_{i}\right) \nu\left(H_{i}\right): M \subset U_{1}^{\infty} G_{i} \times H_{i}\right\} .
$$

(See, for example, $\left[5\right.$, p. 265].) It is easy to see that $(\mu \times \nu)^{S} \leq(\mu \times \nu)^{\prime} \leq$

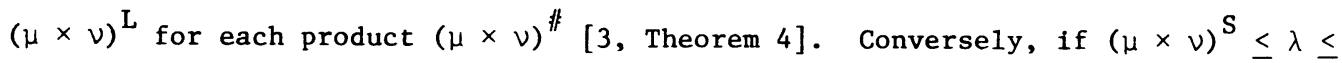
$(\mu \times \nu)^{\mathrm{L}}$, then it is clear that $\lambda$ is itself a product of $\mu$ and $\nu$.

THEOREM 1.1. Suppose $\mu_{1}$ and $\mu_{2}$ are measures on $\delta$ and $\nu$ is a measure on $J$. Then:

$$
\begin{aligned}
& \text { (1) }\left(\left(\mu_{1}+\mu_{2}\right) \times v\right)^{S} \leq\left(\mu_{1} \times v\right)^{S}+\left(\mu_{2} \times v\right)^{S} \leq\left(\mu_{1} \times v\right)^{\mathrm{L}}+\left(\mu_{2} \times v\right)^{\mathrm{L}}= \\
& \left(\left(\mu_{1}+\mu_{2}\right) \times v\right)^{\mathrm{L}} \text {. } \\
& \text { (2) If } \mu_{1} \mathrm{~S} \mu_{2} \text { and } \mu_{2} \mathrm{~S} \mu_{1} \text {, then }\left(\left(\mu_{1}+\mu_{2}\right) \times v\right)^{S}=\left(\mu_{1} \times v\right)^{S}+\left(\mu_{2} \times v\right)^{S} \text {. } \\
& \text { (3) If } \mu_{1} \leq \mu_{2} \text {, then }\left(\mu_{1} \times \nu\right)^{\mathrm{L}} \leq\left(\mu_{2} \times \nu\right)^{\mathrm{L}} \text {. } \\
& \text { (4) }\left(\mu_{1} \times v\right)^{\mathrm{L}} \wedge\left(\mu_{2} \times \nu\right)^{\mathrm{L}}=\left(\left(\mu_{1} \wedge \mu_{2}\right) \times \nu\right)^{\mathrm{L}} \text {. }
\end{aligned}
$$

PROOF. To prove (1) notice that the four measures agree on measurable rectangles $F \times G$ such that $\left(\mu_{1}(F)+\mu_{2}(F)\right) \cup(G)$ is finite. Hence, they agree on measurable subsets of such rectangles and on countable disjoint unions of measurable subsets of such rectangles. Hence, $\left(\left(\mu_{1}+\mu_{2}\right) \times v\right)^{\mathrm{L}}(\mathrm{H})=$ 
$\left(\mu_{1} \times \nu\right)^{\mathrm{L}}(\mathrm{H})+\left(\mu_{2} \times \nu\right)^{\mathrm{L}}(\mathrm{H})$ if the right side is finite. The remaining inequalities are evident.

To prove (2), it suffices to prove $\left(\mu_{1} \times \nu\right)^{S}+\left(\mu_{2} \times \nu\right)^{S} \leq\left(\left(\mu_{1}+\mu_{2}\right) \times \nu\right)^{S}$. Let $H \in S \times$ J. If $k_{1}<\left(\mu_{1} \times \nu\right) S(H)$ and $k_{2}<\left(\mu_{2} \times \nu\right)^{S}(H)$, it suffices to show that $k_{1}+k_{2}<\left(\left(\mu_{1}+\mu_{2}\right) \times \nu\right) S(H)$. Choose measurable rectangles $F_{i} \times G_{1}$ such that $\left(\mu_{i} \times v\right){ }^{S}\left(F_{i} \times G_{i}\right)<\infty$ and $k_{i}<\left(\mu_{i} \times \nu\right) S\left(H \cap\left(F_{i} \times G_{i}\right)\right)$ for $i=1,2$. Since $u_{1} S \mu_{2}$ and $\mu_{2} S \mu_{1}$, we may assume that $F_{1}$ and $F_{2}$ are disjoint and that $\mu_{1}\left(F_{2}\right)=\mu_{2}\left(F_{1}\right)=0$. Then $\mu_{1}$ and $\left(\mu_{1}+\mu_{2}\right)$ agree on $F_{1}$, so that $\mathrm{k}_{1}<\left(\left(\mu_{1}+\mu_{2}\right) \times \nu\right) \mathrm{S}\left(\mathrm{H} \cap\left(\mathrm{F}_{1} \times \mathrm{G}_{1}\right)\right)$. Similarly, $\mathrm{k}_{2}<\left(\left(\mu_{1}+\mu_{2}\right) \times v\right)^{\mathrm{S}}$ $\left(H \cap\left(F_{2} \times G_{2}\right)\right)$. If follows that $k_{1}+k_{2}<\left(\left(\mu_{1}+\mu_{2}\right) \times \nu\right)$ S $(H)$.

Statement (3) follows from the fact that $\left(\mu_{1} \times \nu\right)^{\mathrm{L}}(\mathrm{F} \times \mathrm{G}) \leq\left(\mu_{2} \times \nu\right)^{\mathrm{L}}(\mathrm{F} \times \mathrm{G})$ for all measurable rectangles $F \times G$. To prove $(4)$, notice that $((\mu \wedge \lambda) \times \nu)^{\mathrm{L}} \leq$ $(\mu \times \nu)^{\mathrm{L}} \wedge(\lambda \times \nu)^{\mathrm{L}}$ since $((\mu \wedge \lambda) \times \nu)^{\mathrm{L}}$ is less than or equal to both $(\mu \times \nu)^{\mathrm{L}}$ and $(\lambda \times \nu)^{\mathrm{L}}$. To prove the reverse inequality, it suffices to show that $\left((\mu \times \nu)^{\mathrm{L}} \wedge(\lambda \times \nu)^{\mathrm{L}}\right)(\mathrm{F} \times \mathrm{G}) \leq((\mu \times \lambda) \times \nu)^{\mathrm{L}}(\mathrm{F} \times \mathrm{G})$ if $\mathrm{F} \times \mathrm{G}$ is a measurable rectangle. If $((\mu \times \lambda) \times \nu)^{\mathrm{L}}(\mathrm{F} \times \mathrm{G})=\infty$ or if $\nu(G)=0$, we are done. Hence, we may assume that $(\mu \times \lambda)(F)<\infty$. Then there exists a measurable set $F_{1}$ contained in $F$ such that $\mu \wedge \lambda$ and $\mu$ agree on measurable sets of $F_{1}$ and such that $\mu \wedge \lambda$ and $\lambda$ agree on measurable subsets of $F \backslash F_{1}$. If follows that $((\mu \wedge \lambda) \times \nu)^{\mathrm{L}}\left(\mathrm{F}_{1} \times \mathrm{G}\right)=(\mu \times \nu)^{\mathrm{L}}\left(\mathrm{F}_{1} \times G\right) \geq\left((\mu \times \nu)^{\mathrm{L}} \wedge(\lambda \times \nu)^{\mathrm{L}}\right)\left(\mathrm{F}_{1} \times \mathrm{G}\right)$. Similarly, $((\mu \times \lambda) \times \nu)^{\mathrm{L}}\left(\left(\mathrm{F} \backslash \mathrm{F}_{1}\right) \times \mathrm{G}\right) \geq\left((\mu \times \nu)^{\mathrm{L}} \wedge(\lambda \times \nu)^{\mathrm{L}}\right)\left(\left(\mathrm{F} \backslash \mathrm{F}_{1}\right) \times \mathrm{G}\right)$. Hence, $((\mu \wedge \lambda) \times \nu)^{\mathrm{L}}(\mathrm{F} \times \mathrm{G}) \geq\left((\mu \times \nu)^{\mathrm{L}} \wedge(\lambda \times \nu)^{\mathrm{L}}\right)(\mathrm{F} \times \mathrm{G})$ and we are done.

\section{ABSOLUTE CONTINUITY AND PRODUCT MEASURES.}

THEOREM 2.1. Suppose $\mu \ll \mu^{\prime}$ and $\nu \ll \nu^{\prime}$. Suppose, moreover, that $\mu[$ resp., $\nu]$ is semifinite (and hence, $\sigma$-finite) on any set for which $\mu^{\prime}[$ resp., $\left.\nu^{\prime}\right]$ is finite. Then $(\mu \times \nu)^{\#} \ll\left(\mu^{\prime} \times \nu^{\prime}\right)^{\mathrm{L}}$ for any product $(\mu \times \nu)^{\#}$.

PROOF. Suppose $\left(\mu^{\prime} \times \nu^{\prime}\right)^{L}(M)=0$ and that $M \subset G \times H$, where $\mu^{\prime}(G) \nu^{\prime}(H)<\infty$. It suffices to show that $(\mu \times \nu)^{\mathrm{L}}(\mathrm{M})=0$ in this case. We may assume that $\mu^{\prime}(G)$ and $\nu^{\prime}(H)$ are both positive and finite since $(\mu \times \nu)^{L}(G \times H)=0$ if $\mu^{\prime}(G)=0$ or $\nu^{\prime}(H)=0$. By hypothesis, $\mu$ and $\nu$ are then semifinite and hence o-finite on $G$ and $H$, respectively. It follows from Fubini's theorem that 
$\nu^{\prime}\left(M_{x}\right)=0$ a.e. with respect to $\mu^{\prime}\left[2\right.$, Theorem 36A]. Hence, $\nu\left(M_{x}\right)=0$ a.e. with respect to $\mu$. Then, since $\mu$ and $\nu$ are $\sigma$-finite on $G$ and $H$, respectively, we may apply Fubini's theorem again to say that $(\mu \times \nu)^{L}(M)=\int \nu\left(M_{x}\right) d=0$.

Theorem 2.1 requires the hypothesis that $\mu[$ resp., $\nu]$ be semifinite whenever $\mu^{\prime}\left[\right.$ resp., $\left.\nu^{\prime}\right]$ is finite. For, if $\lambda$ is Lebesgue measure on the unit interval and $\mu$ is $\infty \lambda$, we have $\mu \ll \lambda$, but it is false that $(\mu \times \nu)^{\mathrm{L}} \ll(\lambda \times \lambda)^{\mathrm{L}}$. For, $(\lambda \times \lambda)^{L}$ is 0 on the diagonal, whereas $(\lambda \times \lambda)^{L}$ is $\infty$ on the diagonal.

THEOREM 2.2. Suppose $\mu \ll \mu^{\prime}$, and suppose $\nu(H)=0$ whenever $\nu^{\prime}(H)$ is finite. If $(\mu \times \nu)^{\#}$ is any product of $\mu$ and $\nu$, then $(\mu \times \nu)^{\#}(M)=0$ whenever $\left(\mu^{\prime} \times \nu^{\prime}\right)^{\mathrm{L}}(\mathrm{M})$ is finite. In particular $\left(\mu \times \nu^{\prime \prime}\right)^{k} \ll\left(\mu^{\prime} \times \nu^{\prime}\right)^{\mathrm{L}}$.

PROOF. Suppose $\mu^{\prime}(G) \nu^{\prime}(H)$ is finite. Either $\mu^{\prime}(G)=0$ so that $\mu(G)=0$, or $\mu^{\prime}(G)>0$ so that $\nu^{\prime}(H)$ is finite and $\nu(H)=0$. Hence, $\mu(G) \nu(H)=0$, and it follows that $(\mu \times \nu)^{\#}(M)=0$ whenever $\left(\mu^{\prime} \times \nu^{\prime}\right)^{L}(M)$ is finite.

Let $\mu_{s}$ denote the smallest (semifinite) measure agreeing with $\mu$ on the sets for which $\mu$ is finite (cf. [5, Exercise 11.6b]). It is easy to see that $(\mu \times \nu)^{S}=\left(\mu_{s} \times \nu_{s}\right)^{S}$. The next theorem can be thought of as a dual to Theorem 2.1.

THEOREM 2.3 If $\mu_{s} \ll \mu^{\prime}$, and $\nu_{s} \ll \mu^{\prime}$, then $(\mu \times \nu)^{S} \ll\left(\mu^{\prime} \times \nu^{\prime}\right)^{\#}$ for

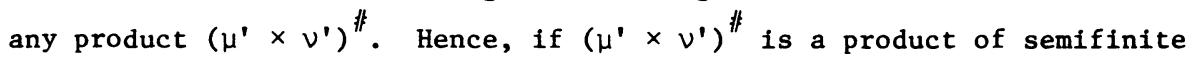
measures $\mu^{\prime}$ and $\nu^{\prime}$ and if $\mu^{\prime} \ll \mu^{\prime}$ and $\nu^{\prime} \ll \nu^{\prime}$, then $(\mu \times \nu)^{S} \ll\left(\mu^{\prime} \times \nu^{\prime}\right)^{\prime \prime}$.

PROOF. Since $\left(\mu^{\prime} \times \nu^{\prime}\right)^{S} \leq\left(\mu^{\prime} \times \nu^{\prime}\right)^{\#}$, it suffices to show that $(\mu \times \nu)^{S} \ll\left(\mu^{\prime} \times \nu^{\prime}\right)^{S}$. Assuming $(\mu \times \nu)^{S}(M)>0$, we show that $\left(\mu^{\prime} \times \nu\right)^{S}(M)>0$. Choose measurable sets $G$ and $H$ such that $\mu(G)$ and $\nu(H)$ are finite and $(\mu \times \nu)^{S}(M \cap(G \times H))>0$. Let $N=M \cap(G \times H)$, and let $A=\left\{x \in G: \nu\left(N_{x}\right)>0\right\}$. Since $\int \nu\left(N_{\mathbf{x}}\right) \mathrm{d} \mu=(\mu \times \nu){ }^{\mathrm{S}}(\mathrm{N})>0$, we have $0<\mu(\mathrm{A}) \leq \mu(G)$. Since $\mu_{\mathbf{s}}(\mathrm{A})=\mu(\mathrm{A})$ and since $\mu_{S} \ll \mu^{\prime}{ }_{S}$, we have $\mu_{S}^{\prime}(A)>0$. Hence, there exists a measurable set B contained in A such that $0<\mu^{\prime}(B)<\infty$. Then $\left(\mu^{\prime} \times \nu\right)^{S}(M) \geq\left(\mu^{\prime} \times \nu\right)^{S}$ $(N \cap(B \times H))=\int_{B} \nu\left(N_{X}\right) d \mu^{\prime}>0$, so that $(\mu \times \nu)^{S} \ll\left(\mu^{\prime} \times \nu\right)^{S}$. Similarly, $\left(\mu^{\prime} \times \nu\right)^{S} \ll\left(\mu^{\prime} \times \nu^{\prime}\right)^{S}$, so that $(\mu \times \nu)^{S} \ll\left(\mu^{\prime} \times \nu^{\prime}\right)^{\#}$.

In Theorem 2.3, we cannot replace the hypotheses that $\mu_{s} \ll \mu_{s}^{\prime}$ and 
$\nu_{s} \ll \nu_{s}^{\prime}$ by $\mu \ll \mu^{\prime}$ and $\nu \ll \nu^{\prime}$ since $\left(\mu^{\prime} \times \nu^{\prime}\right)^{S}$ is 0 if $\mu^{\prime}$ or $\nu^{\prime}$ is degenerate.

In view of [6, Theorem 3.1], we have the following corollary to Theorem 2.3. Although Corollary 2.4 is completely expected, its proof is surprisingly complicated without the use of Theorem 2.3 .

COROLLARY 2.4. Suppose $\mu_{1}$ and $\mu_{2}$ are semifinite measures on $g$ and $\nu$ is a measure on $J$. Then $\left(\left(\mu_{1}+\mu_{2}\right) \times v\right)^{S}=\left(\mu_{1} \times v\right)^{S}+\left(\mu_{2} \times \nu\right)^{S}$.

\section{WEAK SINGULARITY AND PRODUCT MEASURES}

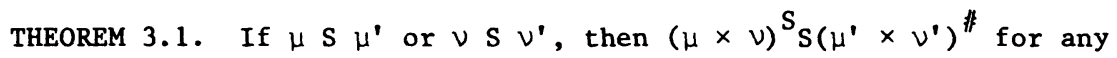
product $\left(\mu^{\prime} \times \nu^{\prime}\right)^{\#}$. Hence, if $\mu$ S $\mu^{\prime}\left(\right.$ or $\nu$ S $\left.\nu^{\prime}\right)$ and $(\mu \times \nu)^{L}$ is $\sigma$-finite, then $(\mu \times \nu)^{\mathrm{L}} \mathrm{S}\left(\mu^{\prime} \times \nu^{\prime}\right)^{\#}$ for any product $\left(\mu^{\prime} \times \nu^{\prime}\right)^{\#}$.

PROOF. Suppose $M$ is a measurable set in the product space, and suppose $\mu S \mu^{\prime}$. Then $(\mu \times \nu)^{S}(M)=(\mu \times \nu)^{S}(M \cap(G \times H)$ for some $G$ and $H$ such that $\mu(G)$ and $\nu(H)$ are $\sigma$-finite. Since $\mu S \mu^{\prime}$ and since $\mu(G)$ is $\sigma$-finite, there exists $G_{1}$ such that $\mu\left(G \backslash G_{1}\right)=0$ and $\mu^{\prime}\left(G_{1}\right)=0[8$, Theorem 3.2]. It follows that $(\mu \times \nu)^{S}(M)=(\mu \times \nu)^{S}\left(M \cap\left(G_{1} \times H\right)\right)$ and $\left(\mu^{\prime} \times \nu^{\prime}\right)^{\prime ⿰}\left(G_{1} \times H\right)=0$. Hence, $(\mu \times \nu)^{S} S\left(\mu^{\prime} \times \nu^{\prime}\right)^{\#}$.

There is no analogue of Theorem 3.1 for largest products; the next example shows that $\mu \mathrm{S} \mu^{\prime}$ does not imply $(\mu \times \nu)^{\mathrm{L}} \mathrm{S}\left(\mu^{\prime} \times \nu\right)^{\mathrm{L}}$.

EXAMPLE 3.2. Let $A$ be a (nonmeasurable) subset of $X=[0,1]$ such that $A$ and $\mathrm{X} \backslash \mathrm{A}$ have Lebesgue outer measure 1 [2, Theorem 16.E]. If $\mathrm{E}$ is a Borel set of $X$, let $\mu(E)$ be the number of points in $E \cap A$ and $\mu^{\prime}(E)$ be the number of points in $E \backslash A$. It is easy to see that $\mu S \mu^{\prime}$. Now, if $D$ is the diagonal of $\mathrm{X} \times \mathrm{X}$, it can be shown that $\left(\mu^{\prime} \times \mu^{\prime}\right)^{\mathrm{L}}(\mathrm{D} \cap \mathrm{F})=\infty$ whenever $\left(\mu \times \mu^{\prime}\right)^{\mathrm{L}}(\mathrm{D} \cap \mathrm{F})>0$. It follows that $\left(\mu \times \mu^{\prime}\right)^{\mathrm{L}}$ is not weakly singular with respect to $\left(\mu^{\prime} \times \mu^{\prime}\right)^{\mathrm{L}}$. Indeed, $\left(\mu \times \mu^{\prime}\right)^{\mathrm{L}}$ is not even weakly singular with respect to $\left(\mu^{\prime} \times \mu^{\prime}\right)^{S}$. Nor does $\left(\mu \times \mu^{\prime}\right)^{L} s\left(\mu^{\prime} \times \mu\right)^{L}$, even though $\mu S \mu^{\prime}$ and $\mu^{\prime} s \mu$.

4. PRODUCT MEASURES AND THE LEBESGUE DECOMPOSITION

In view of the Lebesgue Decomposition Theorem (see below), we shall see

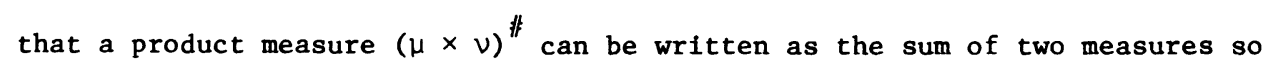
that the first is absolutely continuous with respect to a product measure 


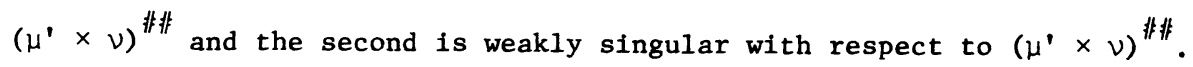
If $\mu^{\prime}$ and $\nu^{\prime}$ are semifinite, we show that the first measure in this decomposition is itself a product measure. For suitable products, we show that the second measure is also a product measure.

Lebesgue Decomposition Theorem ([8, Theorem 2.1]). Suppose $\lambda$ and $\lambda^{\prime}$ are measures on the same sigma-ring. Then $\lambda$ can be written as $\lambda_{\text {ac }}+\lambda_{\text {ws }}$, where $\lambda_{\text {ac }} \ll \lambda^{\prime}$ and $\lambda_{\text {ws }}$ s $\lambda^{\prime}$. The measure $\lambda_{\text {ws }}$ is unique.

The measure $\lambda_{\text {ac }}$ given by the Lebesgue Decomposition Theorem will be called an absolutely continuous part of $\lambda$ with respect to $\lambda^{\prime}$; the measure $\lambda_{\text {ws }}$ will be called the weakly singular part of $\lambda$ with respect to $\lambda^{\prime}$. If $\lambda^{\prime}$ is understood, we shall simply call $\lambda_{\text {ac }}$ an absolutely continuous part of $\lambda$ and $\lambda_{\text {ws }}$ the weakly singular part of $\lambda$.

If $\lambda_{a c} S(E)=\sup \left\{\lambda(E \cap F): \lambda_{w S}(F)=0\right\}$ for each measurable set $E$, then $\lambda_{\mathrm{ac}} \mathrm{S}$ is the smallest absolutely continuous part of $\lambda$ and $\lambda_{\mathrm{ac}} \mathrm{S}$ is weakly singular with respect to $\lambda_{\text {ws }}$. If $\lambda_{\text {ws }}{ }^{L}=\lambda \wedge\left(\infty \lambda^{\prime}\right)$, then $\lambda_{\text {ac }}{ }^{L}$ is the largest absolutely continuous part of $\lambda$ since a measure is absolutely continuous with respect to $\lambda^{\prime}$ if and only if it is less than or equal to $\infty \lambda^{\prime}$. In the reverse direction, if $\lambda_{a c} S \leq \lambda_{1} \leq \lambda_{a c}$, then $\lambda_{1}$ is an absolutely continuous part of $\lambda$. If $\lambda_{a c}{ }^{L}(G)$ is finite, notice that $\lambda_{a c}{ }^{L}(G)=\lambda_{1}(G)$ for each $\lambda^{\prime}$-absolutely continuous part $\lambda_{1}$ of $\lambda$. Therefore, just as all product measures $(\mu \times \nu)^{\# ⿰}$ agree on sets for which the largest product $(\mu \times \nu)^{L}$ is finite, so all $\lambda^{\prime}$-absolutely continuous parts of a measure $\lambda$ agree on sets for which $\lambda_{a c} L$ is finite. Both $\lambda_{a c} \mathrm{~S}$ and $\lambda_{a c} \mathrm{~L}$ behave well with respect to smallest products, as we now show.

LEMMA 4.1. Suppose $\mu$ and $\mu^{\prime}$ are measures on the same sigma-ring $\$$, that $\mu_{a c}$ and $\mu_{a c} L$ are the smallest and largest $\mu^{\prime}$-absolutely continuous parts of $\mu$, that $\mu_{1}$ is any $\mu^{\prime}$-absolutely continuous part of $\mu$, and that $\nu$ is a measure on a sigma-ring $J$. Then:

(i) $\left(\mu_{\text {ac }} \mathrm{S} \times v\right)^{\mathrm{S}} \leq\left(\mu_{1} \times v\right)^{\mathrm{S}}$

and

(ii) $\quad\left(\mu_{\text {ac }}{ }^{L} \times \nu\right)^{S} \leq\left(\mu_{1} \times v\right)^{S}$. Hence, $\left(\mu_{a c}{ }^{S} \times \nu\right)^{S}=\left(\mu_{a c}{ }^{L} \times \nu\right)^{S}$. 
PROOF. Suppose $M \in S \times J$ and that $k<\left(\mu_{\text {ac }} S \times v\right)^{S}(M)$. Then there exist $F$ in $g$ and $G$ in $J$ such that $\mu_{\text {ac }} S(F)<\infty$ and $\nu(G)<\infty$ and such that $k<\left(\mu_{a c} S \times \nu\right) S(M \cap(F \times G))$. Since $\mu_{a c} S S \mu_{w s}$, there exists $F_{1}$ in $g$ such that $\mu_{a c} S^{S}(F)=\mu_{a c} S\left(F_{1}\right)$ and $\mu_{w S}\left(F_{1}\right)=0$. Then $\mu_{a c} S\left(F \backslash F_{1}\right)=0$ since $\mu_{a c} S(F)<\infty$ and $k<\left(\mu_{a c} S \times \nu\right) S\left(M \cap\left(F_{1} \times G\right)\right)$

$$
\begin{aligned}
& \leq\left(\mu_{1} \times v\right)^{S}\left(M \cap\left(F_{1} \times G\right)\right) \\
& \leq\left(\mu_{1} \times v\right)^{S}(M) .
\end{aligned}
$$

Therefore, $\left(\mu_{\text {ac }} \mathrm{S} \times \nu\right)^{\mathrm{S}}(\mathrm{M}) \leq\left(\mu_{1} \times \nu\right)^{\mathrm{S}}(\mathrm{M})$, and we have $\left(\mu_{\text {ac }} \mathrm{S} \times \nu\right)^{\mathrm{S}} \leq\left(\mu_{1} \times \nu\right)^{\mathrm{S}}$. In order to prove (ii), suppose $M \in \delta \times J$ and that $k<\left(\mu_{\text {ac }} L \times \nu\right)$ S $(M)$. Then there exist $F$ in $\delta$ and $G$ in $J$ such that $\mu_{a c} L(F)<\infty$ and $\nu(G)<\infty$ and such that $k<\left(\mu_{a c} L \times \nu\right) S(M \cap(F \times G))$. Since $\mu_{a c} L(F)<\infty$, we have $\mu_{a c} L(F)=\mu_{1}(F)$. Indeed, $\mu_{a c}{ }^{L}\left(F_{1}\right)=\mu_{1}\left(F_{1}\right)$ for all measurable subsets $F_{1}$ of $F$.

Hence,

$$
\begin{aligned}
\mathrm{k} & <\left(\mu_{\mathrm{ac}}{ }^{\mathrm{L}} \times \mathrm{v}^{\mathrm{S}}(\mathrm{M} \cap(\mathrm{F} \times \mathrm{G}))\right. \\
& =\left(\mu_{1} \times \nu\right)^{\mathrm{S}}(\mathrm{M} \cap(\mathrm{F} \times \mathrm{G})) \\
& \leq\left(\mu_{1} \times \nu\right)^{\mathrm{S}}(\mathrm{M}) .
\end{aligned}
$$

Therefore, $\left(\mu_{\text {ac }}{ }^{\mathrm{L}} \times \nu\right)^{\mathrm{S}}(\mathrm{M}) \leq\left(\mu_{1} \times \nu\right)^{\mathrm{S}}(\mathrm{M})$, and we have $\left(\mu_{\mathrm{ac}} \mathrm{L} \times \nu\right)^{\mathrm{S}} \leq\left(\mu_{1} \times \nu\right)^{\mathrm{S}}$.

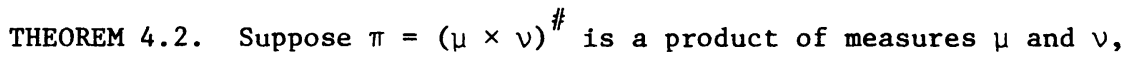
that $\left(\mu^{\prime} \times \nu^{\prime}\right)^{\text {非 }}$ is a product of semifinite measures $\mu^{\prime}$ and $\nu^{\prime}$, and that $\nu \ll \nu^{\prime}$. Let $\mu_{\mathrm{ac}}{ }^{\mathrm{L}}$ be the largest $\mu^{\prime}$-absolutely continuous part of $\mu$, and let

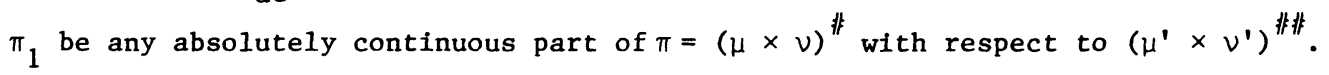
Then $\pi_{1}=\left(\mu_{a c} L \times \nu\right)^{\#}$ for some product of $\mu_{a c} L$ and $\nu$.

PROOF. Assuming $\mu_{a c}{ }^{L}(G) \nu(H)$ is finite, we wish to show that $\pi_{1}(G \times H)=\mu_{a c} L(G) \nu(H)$. If $\nu(H)=0$, then $\pi_{1}(G \times H) \leq \pi(G \times H)=0=\mu_{a c} L(G) \nu(H)$ Hence, we may assume that $\nu(H)>0$ and that $\mu_{a c} L_{(G)}$ is finite. In that case, there exists measurable $G_{1} \subset G$ such that $\mu_{a c} L_{(G)}\left(\mu_{\left(G_{1}\right)}\right.$ and $\mu^{\prime}\left(G \backslash G_{1}\right)=0$. Then $\pi_{1}(G \times H)=\pi_{1}\left(G_{1} \times H\right)$ since $\pi_{1}\left(\left(G \backslash G_{1}\right) \times H\right)=0$. Since $\mu \ll \mu^{\prime}$ on $G_{1}$ and

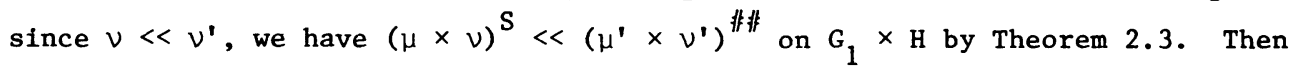
since $\pi_{\text {wS }} \leq \pi$ and since $\pi$ agrees with $(\mu \times \nu)^{S}$ on $G_{1} \times H$, we have

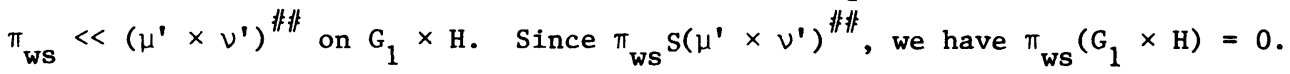


Hence.

$$
\pi_{1}(G \times H)=\pi_{1}\left(G_{1} \times H\right)=\pi\left(G_{1} \times H\right)=\mu\left(G_{1}\right) \nu(H)=\mu_{a c} L^{L}(G) \nu(H),
$$

and we are done.

In Theorem 4.2 we saw conditions under which an absolutely continuous part of a product measure is itself a product measure. We now look at conditions under which the weakly singular part of $(\mu \times \nu){ }^{\#}$ with respect to

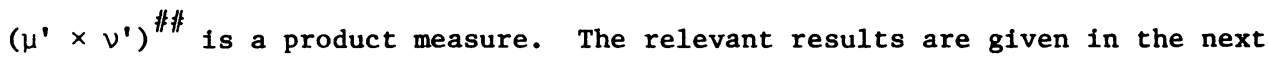
theorem.

THEOREM 4.3. Suppose $\pi=(\mu \times \nu)^{\#}$ is a product of measures $\mu$ and $\nu$, that

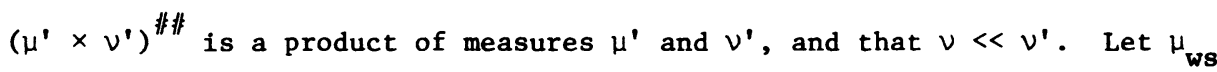
be the weakly singular part of $\mu$ with respect to $\mu^{\prime}$, and let $\pi_{w s}$ be the weakly singular part of $\pi=(\mu \times \nu)^{\#}$ with respect to $\left(\mu^{\prime} \times \nu^{\prime}\right)^{\# \#}$. Then:

(1) $\left(\mu_{w s} \times \nu\right)^{S} \leq \pi_{w s}$.

(2) If $\pi$ is the smallest product of $\mu$ and $\nu$ and if $\mu^{\prime}$ and $\nu^{\prime}$ are semifinite, then $\pi_{w s}=\left(\mu_{w s} \times \nu\right)^{S}$.

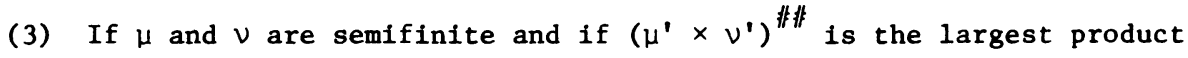
of $\mu^{\prime}$ and $\nu^{\prime}$, then $\pi_{w s}$ is a product of $\mu_{w s}$ and $\nu$.

PROOF. Let $\pi_{1}$ be any absolutely continuous part of $\pi$ with respect

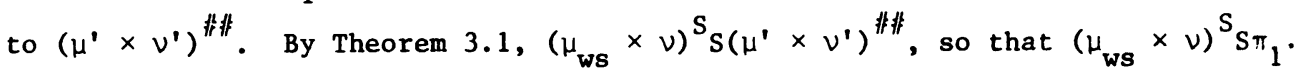
Since $\left.\left(\mu_{w s} \times \nu\right)^{S} \leq i \mu \times \nu\right)^{S} \leq \pi=\pi_{1}+\pi_{w s^{\prime}}$ we therefore have $\left(\mu_{w s} \times \nu\right)^{S} \leq \pi_{w s}$ $[8$, p. 628].

In order to prove (2), let $\mu_{1}$ be any $\mu^{\prime}$-absolutely continuous part of $\mu$ and notice that $(\mu \times \nu)^{S} \leq\left(\mu_{1} \times \nu\right)^{S}+\left(\mu_{w s} \times \nu\right)^{S}$ by Theorem 1.1(1). We have

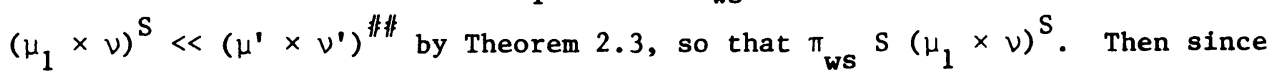
$\pi_{w s} \leq\left(\mu_{1} \times \nu\right)^{S}+\left(\mu_{w s} \times \nu\right)^{S}$, we have $\pi_{w s} \leq\left(\mu_{w s} \times \nu\right)^{S}$. We already know from (1) that $\left(\mu_{w s} \times v\right)^{S} \leq \pi_{w s}$, so that $\pi_{w s}=\left(\mu_{w s} \times v\right)^{S}$ in this case.

In order to prove $(3)$, it suffices to show that $\pi_{w s} \leq\left(\mu_{w s} \times \nu\right)^{L}$ since we already know that $\left(\mu_{\text {ws }} \times \nu\right)^{S} \leq \pi_{\text {ws }}$. Let $\mu_{\text {ac }}$ S be the smallest $\mu^{\prime}$-absolutely continuous part of $\mu$. Since $\mu$ is semifinite, so is $\mu_{\text {ac }} S$. Then $\left(\mu_{a c} \mathrm{~S} \times \nu\right)^{\mathrm{L}} \ll\left(\mu^{\prime} \times \nu^{\prime}\right)$ by Theorem 2.1 , so that $\pi_{w s} \mathrm{~S}\left(\mu_{a c} \mathrm{~S} \times \nu\right)^{\mathrm{L}}$. Since $\pi_{w s} \leq(\mu \times \nu)^{\#} \leq(\mu \times \nu)^{\mathrm{L}}=\left(\mu_{\mathrm{ac}} \mathrm{S} \times \nu\right)^{\mathrm{L}}+\left(\mu_{\text {ws }} \times \nu\right)^{\mathrm{L}}$, 
we have $\pi_{\text {ws }} \leq\left(\mu_{\text {ws }} \times v\right)^{L}$, as was to be shown.

We pause to observe that the measure $\pi_{\text {ws }}$ is Theorem 4.3 need not always be a product measure. Nor does it help to assume that all measures under consideration are semifinite measures.

EXAMPLE 4.4. Let $\mu$ be counting (discrete) measure on the Borel sets $\delta$ of the unit interval, and let $v$ be Lebesgue measure on $\delta$. Define a measure $\lambda$ on $\delta \times S$ by

$$
\lambda(M)=v(\{y \in Y:(y, y) \in M\}) \text { if } M \in \delta \times \delta .
$$

In other words, $\lambda(M)$ is found by intersecting $M$ with the diagonal and using Lebesgue measure of the projection of that trace. Let $\pi=(\mu \times \nu)^{S}+\lambda$. It is easy to see that $\pi$ is a product of $\mu$ and $\nu$ since $\lambda(G \times H)=0$ if $(\mu \times \nu) S(G \times H)<\infty$. If $\pi_{\text {wS }}$ is the weakly singular part of $\pi$ with respect to $(\mu \times \nu)^{S}$, then $i t^{\circ}$ is easy to see that $\pi_{w s}=\lambda$. Hence, $\pi_{w s}$ is not a product measure in this case.

For the remainder of the paper, we shall investigate the Lebesgue

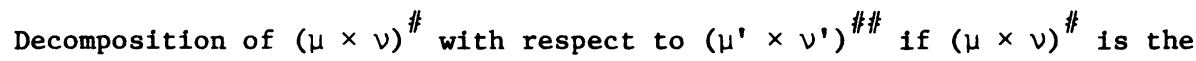
smallest product of $\mu$ and $\nu$ or if the products $(\mu \times \nu)^{\#}$ and $\left(\mu^{\prime} \times \nu^{\prime}\right)^{\# ⿰}$ are largest products.

THEOREM 4.5. Suppose $\pi=(\mu \times \nu)^{S}$ is the smallest product of measures $\mu$ and $\nu$, that $\left(\mu^{\prime} \times \nu^{\prime}\right)^{\#}$ is a product of semifinite measures $\mu^{\prime}$ and $\nu^{\prime}$, and that $\nu \ll \nu^{\prime}$. Let $\mu_{\text {ac }}$ be any $\mu^{\prime}$-absolutely continuous part of $\mu$, let $\pi_{\text {ac }} S_{\text {be }}$ the smallest $\left(\mu^{\prime} \times \nu^{\prime}\right)^{\#}$-absolutely continuous part of $(\mu \times \nu)$, let $\mu_{\text {ws }}$ be the $\mu^{\prime}$-weakly singular part of $\mu$, and let $\pi_{\text {ws }}$ be the $\left(\mu^{\prime} \times \nu^{\prime}\right)^{\prime \prime}$-weakly singular part of $\pi$. Then

(1) $\left(\mu_{a c}^{S} \times \nu\right)^{S}=\left(\mu_{a c}^{L} \times \nu\right)^{S}=\pi_{a c} S$

(2) $\quad\left(\mu_{\text {ws }} \times v\right)^{S}=\pi_{\text {ws }}$.

PROOF. We know that $\left(\mu_{a c}^{S} \times \nu\right)^{S}=\left(\mu_{a c}^{L} \times \nu\right)^{S}$ by Lemma 4.1 and that $\left(\mu_{\text {ac }}^{L} \times \nu\right)^{S} \leq \pi_{\text {ac }}{ }^{S}$ by Theorem 4.2. Moreover $\left(\mu_{w s} \times \nu\right)^{S}=\pi_{w s}$ by Theorem 4.3.

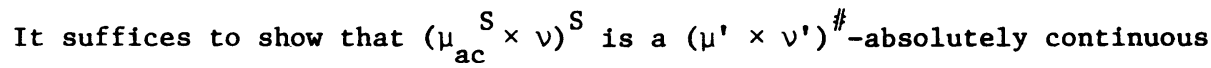
part of $(\mu \times \nu)^{S}$. Now $\left(\mu_{a c}^{S} \times \nu\right)^{S} \ll\left(\mu^{\prime} \times \nu^{\prime}\right)^{\#}$ by Theorem 2.3, and $(\mu \times \nu)^{S}=\left(\mu_{a c} S \times \nu\right)^{S}+\left(\mu_{w s}^{S}+\nu\right)^{S}$ by Theorem 1.1(2). Hence $\left(\mu_{a c} S \times \nu\right)^{S}$ 
is indeed a $\left(\mu^{\prime} \times \nu^{\prime}\right)^{\#}$-absolutely continuous part of $(\mu \times \nu)^{S}$.

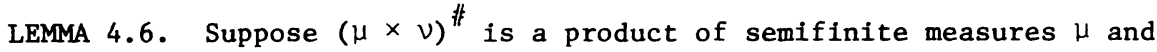
$\nu$. If $\left(\mu^{\prime} \times \nu^{\prime}\right)^{S}$ is $\sigma$-finite and $\mu S \mu^{\prime}$, then $(\mu \times \nu)^{\#} S\left(\mu^{\prime} \times \nu^{\prime}\right)^{S}$. Hence, if $\left(\mu^{\prime} \times \nu^{\prime}\right)^{\mathrm{L}}$ is $\sigma$-finite and $\mu \mathrm{S} \mu^{\prime}$, then $(\mu \times \nu)^{\prime \prime} \mathrm{S}\left(\mu^{\prime} \times \nu^{\prime}\right)^{\mathrm{L}}$.

PROOF. Let $\mu^{\prime}{ }_{\text {ac }} \mathrm{S}$ and $\nu^{\prime}{ }_{\mathrm{ac}}^{\mathrm{S}}$ be the smallest absolutely continuous parts of $\mu^{\prime}$ and $\nu^{\prime}$ with respect to $\mu$ and $\nu$, respectively. Let $\mu^{\prime}{ }_{\text {ws }}$ and $\nu^{\prime}$ ws be the weakly singular parts of $\mu^{\prime}$ and $\nu^{\prime}$ with respect to $\mu$ and $\nu$, respectively. By

Theorem 1.1(2), we have

$$
\left(\mu^{\prime} \times \nu^{\prime}\right)^{\mathrm{S}}=\left(\mu^{\prime}{ }_{\mathrm{ac}}^{\mathrm{S}} \times \nu^{\prime}{ }_{\mathrm{ac}} \mathrm{S}\right)+\left(\mu^{\prime}{ }_{\mathrm{ac}}^{\mathrm{S}} \times \nu^{\prime}{ }_{\text {ws }}\right)+\left(\mu^{\prime}{ }_{\text {ws }} \times \nu^{\prime}\right)^{\mathrm{S}} \text {; }
$$

each of these products is clearly $\sigma$-finite. In view of Theorem 3.1 , we have $\left(\mu^{\prime} \times \nu^{\prime}\right)^{S} S(\mu \times \nu)^{\#}$ and $\left(\mu^{\prime} \times \nu^{\prime}{ }_{\text {ws }}\right)^{S} S(\mu \times \nu)^{S}$, so that $(\mu \times \nu)^{\#} \mathrm{~S}\left(\mu^{\prime}{ }_{\text {ws }} \times \nu^{\prime}\right)^{S}$ and $(\mu \times \nu)^{\#} \mathrm{~S}\left(\mu_{\mathrm{ac}}^{\prime} \mathrm{S} \times \nu^{\prime}{ }_{\text {ws }}\right)^{\mathrm{S}}[8$, Theorem 3.2]. Now $\left(\mu^{\prime}{ }_{\text {ac }} \mathrm{S} \times \nu^{\prime}{ }_{\text {ac }} \mathrm{S}\right) \mathrm{S} \ll(\mu \times \nu)^{\mathrm{S}}$ by Theorem 2.3 , and $(\mu \times \nu) \mathrm{S} S\left(\mu^{\prime}{ }_{\text {ac }}^{\mathrm{S}} \times \nu^{\prime}{ }_{\text {ac }} \mathrm{S}\right) \mathrm{S}$ by Theorem 3.1. Thus, $(\mu \times \nu)^{\#} \mathrm{~S}\left(\mu_{\mathrm{ac}}^{\prime} \mathrm{S} \times \nu_{\text {ac }}^{\prime}{ }^{\mathrm{S}}\right) \mathrm{S}[8$, Theorem $2.4(1 \mathrm{~b})]$. Hence, $(\mu \times \nu)^{\#} \mathrm{~S}\left(\mu^{\prime} \times \nu^{\prime}\right)^{\mathrm{S}}$.

We say that $\nu$ is quasi-dominant with respect to $\nu^{\prime}$ if for each measurable set $E$, there exists a measurable set $F$ such that $\nu(E)=V(E \cap F)$ and such that $\nu^{\prime}{ }_{F}$ is absolutely continuous with respect to $\nu_{F}$ (that is, $\nu^{\prime}(F \cap G)=0$ whenever $\nu(G \cap F)=0)$. For example, if $\nu^{\prime}$ is absolutely continuous with respect to $\nu$ or if $\nu^{\prime}$ is $\sigma$-finite, then $\nu$ is quasi-dominant with respect to $\nu^{\prime}[9$, pp. 118-119].

THEOREM 4.7. Suppose $\mu$ and $\mu^{\prime}$ are semifinite measures on $\delta$, and suppose $\nu$ and $\nu^{\prime}$ are semifinite measures on $J$ such that $\nu$ is both absolutely continuous and quasi-dominant with respect to $\nu^{\prime}$. Let $\mu_{a c}{ }^{S}$ be the smallest $\mu^{\prime}$-absolutely continuous part of $\mu$, let $\mu_{\text {ws }}$ be the $\mu^{\prime}$-weakly singular part of $\mu$, and let $\mu_{1}=\mu_{a c} S+\left(\mu_{w s} \wedge \mu^{\prime}\right)$. Then $\left(\mu_{1} \times \nu\right)^{L}$ is an absolutely continuous part of $\pi=(\mu \times \nu)^{L}$ with respect to $\left(\mu^{\prime} \times \nu^{\prime}\right)^{L}$.

PROOF. Evidently, $\mu_{\text {ac }} S \leq \mu_{\text {ac }} S+\left(\mu_{\text {ws }} \wedge \mu^{\prime}\right) \leq \mu \wedge$ o $\mu^{\prime}=\mu_{a c}{ }^{L}$, so that $\mu_{1}$ is a $\mu^{\prime}$-absolutely continuous part of $\mu$. Then $\left(\mu_{1} \times \nu\right)^{\mathrm{L}}$ is absolutely continuous with respect to $\left(\mu^{\prime} \times \nu^{\prime}\right)^{\mathrm{L}}$ by Theorem 2.1. Then by Theorem 1.1 (1) and Theorem 4.3, we have $(\mu \times \nu)^{\mathrm{L}}=\left(\mu_{1} \times \nu\right)^{\mathrm{L}}+\left(\mu_{\text {ws }} \times \nu\right)^{\mathrm{L}} \geq\left(\mu_{1} \times \nu\right)^{\mathrm{L}}+\pi_{\text {ws }}$. 
In order to prove the reverse inequality, we first show that $\left(\mu_{w s} \times \nu\right)^{L}(M)=0$ if $\left(\mu^{\prime} \times \nu\right)^{\mathrm{L}}(M)$ is finite and $\pi_{w s}(M)=0$. Assuming $\left(\mu^{\prime} \times \nu\right)^{\mathrm{L}}(M)$ is finite and $\pi_{w S}(M)=0$, we may assume without loss of generality that $M$ is a subset of $G \times H$, where $\mu^{\prime}(G)$ and $\nu(H)$ are finite. Then since $\nu$ is quasi-dominant with respect to $\nu^{\prime}$, we may assume that $\nu^{\prime} \ll \nu$ on $H$. Since $\mu^{\prime}(G)$ is finite, Lemma 4.6 tells us that $\left(\mu_{w S} \times \nu\right)_{G}^{L} \times H$ is weakly singular with respect to $\left(\mu^{\prime} \times v\right)_{\mathrm{G} \times \mathrm{H}^{\text {}}}^{\mathrm{L}}$. In other words, there exists $\mathrm{N} \in \mathrm{S} \times \mathrm{J}$ such that $\left(\mu_{\text {ws }} \times \nu\right)^{\mathrm{L}}(\mathrm{M})=\left(\mu_{\text {wS }} \times \nu\right)^{\mathrm{L}}(\mathrm{M} \cap \mathrm{N})$ and $\left(\mu^{\prime} \times \nu\right)^{\mathrm{L}}(\mathrm{N})=0$. We have $\left(\mu^{\prime} \times \nu^{\prime}\right)^{\mathrm{L}}{ }_{\mathrm{G} \times \mathrm{H}} \ll\left(\mu^{\prime} \times \nu\right)^{\mathrm{L}}{ }_{\mathrm{G} \times \mathrm{H}}$ by Theorem 2.1 , so that $\left(\mu^{\prime} \times \nu^{\prime}\right)^{\mathrm{L}}(\mathrm{N})=0$. Thus

$$
\begin{aligned}
\left(\mu_{w s} \times v\right)^{L}(M) & =\left(\mu_{w s} \times v\right)^{L}(M \cap N) \\
& \leq \pi(M \cap N) \\
& =\pi_{a c}(M \cap N)+\pi_{w s}(M \cap N) \\
& =0+0
\end{aligned}
$$

Hence, $\left(\mu_{\text {ws }} \times v\right)^{\mathrm{L}}(\mathrm{P}) \leq\left(\mu^{\prime} \times \nu\right)^{\mathrm{L}}(\mathrm{P})$ for all measurable subsets $\mathrm{P}$ of $\mathrm{M}$ if $\pi_{w s}(M)=0$. By Theorem $1.1(4)$, we have

$$
\begin{aligned}
\left(\mu_{w s} \times \nu\right)^{L}(P) & \leq\left(\left(\mu_{w s} \times \nu\right)^{L} \wedge\left(\mu^{\prime} \times \nu\right)^{L}\right)(P) \\
& =\left(\left(\mu_{w s} \wedge \mu^{\prime}\right) \times \nu\right)^{L}(P) \\
& \leq\left(\mu_{w s} \times \nu\right)^{L}(P)
\end{aligned}
$$

if $\pi_{w s}(P)=0$. Now if $\pi_{w s}(M)$ is finite, there exists $N \in S \times J$ such that $\left(\mu^{\prime} \times \nu^{\prime}\right)^{L}(N)=0$ and such that $\pi_{w s}(M)=\pi_{w s}(M \cap N)$. Since $\pi_{w s}(M \backslash N)=0$, we have

$$
\begin{aligned}
& (\mu \times \nu)^{\mathrm{L}}(\mathrm{M})=\left(\mu_{\mathrm{ac}}^{\mathrm{S}} \times \nu\right)^{\mathrm{L}}(\mathrm{M} \backslash \mathrm{N})+\left(\mu_{w S} \times \nu\right)^{\mathrm{L}}(\mathrm{M} \backslash \mathrm{N})+\pi(\mathrm{M} \cap \mathrm{N}) \\
& =\left(\mu_{a c} S \times \nu\right)^{L}(M \backslash N)+\left(\left(\mu_{w S} \times \nu^{\prime}\right) \times \nu\right)^{L}(M \backslash N)+\pi_{w S}(M \cap N) \\
& =\left(\mu_{1} \times v\right)^{\mathrm{L}}(\mathrm{M} \backslash \mathrm{N})+\pi_{\text {ws }}(\mathrm{M} \cap \mathrm{N}) \\
& =\left(\mu_{1} \times v\right)^{L}(M)+\pi_{w s}(M) \text {. }
\end{aligned}
$$

We have thus shown that $(\mu \times \nu)^{L}$ is the sum of $\left(\mu_{1} \times \nu\right)^{L}$ and $\pi_{\text {ws }}$, so that $\left(\mu_{1} \times \nu\right)^{\mathrm{L}}$ is indeed an absolutely continuous part of $\pi$.

COROLLARY 4.8. Suppose $\mu$ and $\mu^{\prime}$ are semifinite measures on $\delta$ and that $\nu$ is a semifinite measure on $J$. Let $\mu_{a c} S$ be the smallest $\mu^{\prime}$-absolutely continuous part of $\mu$, let $\mu_{\text {wS }}$ be the $\mu^{\prime}$-weakly singular 
part of $\mu$, and let $\mu_{1}=\mu_{\text {ac }} S^{S}+\left(\mu_{\text {ws }} \wedge \mu^{\prime}\right)$. Then $\left(\mu_{1} \times \nu\right)^{L}$ is an absolutely continuous part of $\pi=(\mu \times \nu)^{\mathrm{L}}$ with respect to $\left(\mu^{\prime} \times \nu\right)^{\mathrm{L}}$.

Let $\pi_{\text {ws }}$ be the weakly singular part of $\pi=(\mu \times \nu)^{\mathrm{L}}$ with respect to $\left(\mu^{\prime} \times \nu\right)^{\mathrm{L}}$. We pause to observe that the conditions of Corollary 4.8 do not imply that $\pi_{w s}=\left(\mu_{w s} \times \nu\right)^{\mathrm{L}}$. Recall that in Example 3.2 we have $\mu=\mu_{\text {ws }}$ and $\pi_{\text {ws }} \neq(\mu \times \nu)^{\mathrm{L}}$ since $\mu^{\mathrm{S}^{\prime}} \mu^{\prime}$ and since $(\mu \times \nu)^{\mathrm{L}}$ is not weakly singular with respect to $\left(\mu^{\prime} \times \nu^{\mathrm{L}}\right.$.

At the opposite extreme from quasi-dominance is strong recessiveness. We say that $\nu$ is strongly recessive with respect to $\nu^{\prime}$, denoted $\nu_{S} v^{\prime}$,

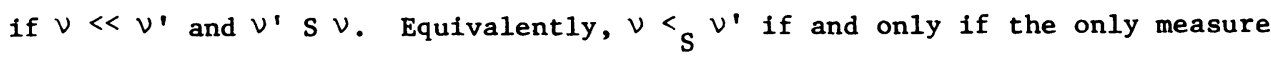
$\lambda$ which is both quasi-dominant with respect to $\nu^{\prime}$ and less than or equal to $\nu$ is $\lambda=0$. If $\nu$ and $\nu^{\prime}$ are measures on the same sigma-ring, then $\nu$ can be written as $\nu_{1}+\nu_{2}$, where $\nu_{1}$ is strongly recessive with respect to $\nu^{\prime}$ and $\nu_{2}$ is quasi-dominant with respect to $\nu^{\prime}[9$, Theorem 2.5]. Whereas Theorem 4.7 dealt with the case where $\nu \ll \nu^{\prime}$ and $\nu$ is quasi-dominant with respect to $\nu^{\prime}$, so Theorem 4.9 tells us what happens if $\nu$ is strongly recessive with respect to $\nu^{\prime}$. For if $\nu_{S} \nu^{\prime}$, notice that $\nu(H)$ vanishes whenever $V^{\prime}(H)$ is finite. THEOREM 4.9. Suppose $\mu$ and $\mu^{\prime}$ are semifinite measures on $\delta$, and suppose $\nu$ and $\nu^{\prime}$ are semifinite measures on $J$ such that $\nu$ vanishes whenever $\nu^{\prime}$ is finite. Let $\mu_{a c}$ L be the largest $\mu^{\prime}$-absolutely continuous part of $\mu$. Then $\left(\mu_{\mathrm{ac}}^{\mathrm{L}} \times \nu\right)^{\mathrm{L}}$ is the largest absolutely continuous part of $\pi=(\mu \times \nu)^{\mathrm{L}}$ with respect to $\left(\mu^{\prime} \times \nu^{\prime}\right)^{\mathrm{L}}$.

PROOF. We know that $\left(\mu_{\mathrm{ac}}^{\mathrm{L}} \times \nu\right)^{\mathrm{L}} \ll\left(\mu^{\prime} \times \nu^{\prime}\right)^{\mathrm{L}}$ by Theorem 2.2. Let $\pi_{\text {ac }}{ }^{\mathrm{L}}$ be the largest absolutely continuous part of $\pi$ with respect to $\left(\mu^{\prime} \times \nu^{\prime}\right)^{\mathrm{L}}$, and let $\pi_{w s}$ be the weakly singular part of $\pi$ with respect to $\left(\mu^{\prime} \times \nu^{\prime}\right)^{L}$. We have $\pi_{a c}{ }^{L} \leq\left(\mu_{a c} L \times \nu\right)^{L}$ by Theorem 4.2, $\pi_{w s} \leq\left(\mu_{w s} \times \nu\right)^{L}$ by Theorem 4.3 (3), and $(\mu \times \nu)^{\mathrm{L}}=\left(\mu_{\mathrm{ac}}^{\mathrm{L}} \times \nu\right)^{\mathrm{L}}+\left(\mu_{\mathrm{ws}} \times \nu\right)^{\mathrm{L}}$ by Theorem 1.1 (1). Hence,

$$
\begin{aligned}
(\mu \times v)^{\mathrm{L}} & =\left(\mu_{\mathrm{ac}}^{\mathrm{L}} \times \nu\right)^{\mathrm{L}}+\left(\mu_{w s} \times \nu\right)^{\mathrm{L}} \\
& \geq\left(\mu_{\mathrm{ac}}^{\mathrm{L}} \times \nu\right)^{\mathrm{L}}+\pi_{w s} \\
& \geq \pi_{\mathrm{ac}} \mathrm{L}+\pi_{w s} \\
& =(\mu \times \nu)^{\mathrm{L}}
\end{aligned}
$$


so that $\left(\mu_{a c} \mathrm{~L} \times \nu\right)^{\mathrm{L}}$ is an absolutely continuous part of $\pi$ with respect to $\left(\mu^{\prime} \times \nu^{\prime}\right)^{L}$. Then since $\left(\mu_{a c}{ }^{L} \times \nu\right)^{L} \leq \pi_{a c}^{L}$, we have $\left(\mu_{a c}{ }^{L} \times \nu\right)^{L}=\pi_{a c}{ }^{L}$. Finally, suppose $\mu$ and $\mu^{\prime}$ are semifinite measures on $\delta$, and suppose $\nu$ and $\nu^{\prime}$ are semifinite measures on $J$ such that $\nu \ll \nu^{\prime}$. Does there exist a measure $\mu_{0}$ such that $\left(\mu_{0} \times \nu\right)^{\mathrm{L}}$ is an absolutely continuous part of $\pi=(\mu \times \nu)^{\mathrm{L}}$ with respect to $\left(\mu^{\prime} \times \nu^{\prime}\right)^{\mathrm{L}}$ ? The answer is no, as Example 4.10 shows.

EXAMPLE 4.10. (cf. $[9, \mathrm{pp} .131-132]$.$) Let \mathrm{X}=[0,1)$, and let $\mathrm{s}$ be the Borel sets of $x$. Let $\mu$ be counting measure on $\delta$, and let $\mu^{\prime}$ be Lebesgue measure on $S$. Let $Y=[0,2)$, let $J$ be the Borel sets of $Y$, and let $\nu$ be Lebesgue measure on $J$. If $H \in J$, let $\nu^{\prime}(H)$ be Lebesgue measure of $H \cap[0,1)$ plus counting measure of $\mathrm{H} \cap[1,2)$. Let $\mathrm{D}=\{(\mathrm{x}, \mathrm{y}) \in \mathrm{X} \times \mathrm{Y}: \mathrm{x}=\mathrm{y}\}$, and let $E=\{x, y) \in X \times Y: y=x+1\}$. Suppose there were a measure $\mu_{0}$ such that $\left(\mu_{0} \times \nu\right)^{\mathrm{L}}$ is an absolutely continuous part of $(\mu \times \nu)^{\mathrm{L}}$ with respect to $\left(\mu^{\prime} \times \nu^{\prime}\right)^{\mathrm{L}}$. We would then have $\left(\mu_{0} \times \nu\right)^{\mathrm{L}}(\mathrm{D})=0$ and $\left(\mu_{0} \times \nu\right)^{\mathrm{L}}(\mathrm{E})=\infty$, which is clearly impossible.

\section{COMPLEMENTS ON STRONG-RECESSIVENESS AND QUASI-DOMINANCE}

THEOREM 5.1. Let $\mu^{\prime}$ and $\nu^{\prime}$ be semifinite measures. Suppose $\mu<_{S} \mu^{\prime}$ and $\nu \ll \nu^{\prime}$, or suppose $\mu \ll \mu^{\prime}$ and $\nu<_{S} \nu^{\prime}$. Then $(\mu \times \nu)^{S}<_{S}\left(\mu^{\prime} \times \nu^{\prime}\right)^{\# !}$ for any product $\left(\mu^{\prime} \times \nu^{\prime}\right)^{\#}$.

PROOF. By Theorem 2.3, $(\mu \times \nu)^{S} \ll\left(\mu^{\prime} \times \nu^{\prime}\right)^{S}$. Then by Theorem 3.1 and by the hypothesis that $\mu^{\prime} \mathrm{s} \mu$ or $\nu^{\prime} \mathrm{s} \nu$, we have $\left(\mu^{\prime} \times \nu^{\prime}\right)^{S} \mathrm{~s}(\mu \times \nu)^{S}$. Hence, $(\mu \times \nu)^{S}<_{S}\left(\mu^{\prime} \times \nu^{\prime}\right)^{S}$. If $\left(\mu^{\prime} \times \nu^{\prime}\right)^{\#}$ is any product of $\mu^{\prime}$ and $\nu^{\prime}$ we observe that $\left(\mu^{\prime} \times \nu^{\prime}\right)^{S} \leq\left(\mu^{\prime} \times \nu^{\prime}\right)^{\#}$ so that $(\mu \times \nu)^{S}<_{S}\left(\mu^{\prime} \times \nu^{\prime}\right)^{\# ~}$ [9, Theorem $2.4(1 \mathrm{~b})]$.

There is no analogue of Theorem 5.1 for largest products. Example 5.2 shows that the conditions $\mu<_{S} \mu^{\prime}$ and $\nu<_{S} \nu^{\prime}$ do not imply $(\mu \times \nu)^{L}<_{S}\left(\mu^{\prime} \times \nu^{\prime}\right)^{L}$.

EXAMPLE 5.2. As in Example 3.2, let A be a (nonmeasurable) subset of $X=[0,1]$ such that $A$ and $B=X \backslash A$ have Lebesgue outer measure 1 . If $E$ is a Borel set of $X$, let $\kappa_{A}(E)$ be the number of points in $E \cap A$ and let $K_{B}(E)$ be the number of points in $E \cap B$. Let $\lambda$ be Lebesgue measure on the Borel 
sets of $x$, and let $\mu=\nu=\infty \lambda$. Clearly, $\mu<_{S} \kappa_{A}$ and $\mu<_{S} \kappa_{B}$. Now, if $D$ is the diagonal of $X \times x$, it can be seen that $\left(K_{A} \times K_{B}\right)^{L}(D)=\infty$ and that $(\mu \times v)^{L}(D \cap M)=\infty$ whenever $\left(\kappa_{A} \times \kappa_{B}\right)^{L}(D \cap M)=\infty$. Hence, $\left(\kappa_{A} \times \kappa_{B}\right)^{L}$ is not weakly singular with respect to $(\mu \times \nu)^{L}$, so that $(\mu \times \nu)^{L}$ is not strongly recessive with respect to $\left(\kappa_{A} \times \kappa_{B}\right)^{L}$. Of course $(\mu \times \nu)^{\mathrm{L}} \ll\left(\kappa_{A} \times \kappa_{B}\right)^{\mathrm{L}}$ by Theorem 2.2.

Let us write $\mu Q \mu^{\prime}$ if $\mu$ is quasi-dominant with respect to $\mu^{\prime}$ (see discussion preceding Theorem 4.7). In Theorem 5.4 we use the fact that $\mu Q \mu{ }^{\prime}$ if and only if there exist measures $\mu^{\prime}{ }_{1}$ and $\mu^{\prime}{ }_{2}$ such that $\mu S \mu^{\prime}{ }_{1}$ and $\mu_{2}^{\prime} \ll \mu$ and $\mu^{\prime}=\mu_{1}^{\prime}+\mu_{2}^{\prime}[9$, Theorem 2.1 (4)]

LEMMA 5.3. If $\mu Q \mu^{\prime}$ and $\mu_{s}$ is the smallest (semifinite) measure agreeing with $\mu$ on sets of finite $\mu$-measure, then $\mu_{s} Q \mu^{\prime}$.

PROOF. Since $\mu_{S}=\sup \left\{\mu_{A}: \mu(A)<\infty\right\}$, the result follows from Theorem 2.1 (8) and Theorem 3.1 of [9].

THEOREM 5.4. Suppose $\mu Q \mu^{\prime}$ and $\nu Q \nu^{\prime}$. Then $(\mu \times \nu)^{S} Q\left(\mu^{\prime} \times \nu^{\prime}\right)^{S}$. PROOF. In view of Lemma 5.3, we have $\mu_{s} Q \mu^{\prime}$ and $\nu_{s} Q \nu^{\prime}$. Then since $(\mu \times \nu)^{S}=\left(\mu_{S} \times \nu_{S}\right)^{S}$, we may assume without loss of generality that $\mu$ and $\nu$ are both semifinite. Since $\mu Q \mu^{\prime}$, there exist measures $\mu^{\prime}{ }_{1}$ and $\mu^{\prime}{ }_{2}$ such that $\mu \mathrm{S} \mu^{\prime}{ }_{1}$ and $\mu_{2}^{\prime} \ll \mu$. Similarly, there exist $\nu^{\prime}{ }_{1}$ and $\nu^{\prime}{ }_{2}$ such that $\nu \mathrm{S} \nu^{\prime}{ }_{1}$ and $\nu^{\prime}{ }_{2} \ll \nu$. Then $(\mu \times \nu)^{S} \mathrm{~S}\left(\mu^{\prime}{ }_{1} \times \nu^{\prime}\right)^{\mathrm{S}}$ and $(\mu \times \nu)^{\mathrm{S}} \mathrm{S}\left(\mu^{\prime}{ }_{2} \times \nu^{\prime}{ }_{1}\right)^{\mathrm{S}}$ by Theorem 3.1, and $\left(\mu_{2}^{\prime} \times \nu^{\prime}{ }_{2}\right)^{S} \ll(\mu \times \nu)^{S}$ by Theorem 2.3. Since $(\mu \times \nu)^{S}$ is weakly sir.ular with respect to $\left(\mu^{\prime}{ }_{1} \times \nu^{\prime}\right)^{S}+\left(\mu_{2}^{\prime} \times \nu^{\prime}{ }_{1}\right)^{S}$ and since $\left(\mu^{\prime}{ }_{2} \times \nu^{\prime}{ }_{2}\right)^{S} \ll(\mu \times \nu)^{S}$, we see that $(\mu \times v)^{S}$ is quasi-dominant with respect to the measure

$$
\rho=\left(\mu^{\prime}{ }_{1} \times \nu^{\prime}\right)^{S}+\left(\mu_{2}^{\prime} \times \nu^{\prime}{ }_{1}\right)^{S}+\left(\mu^{\prime}{ }_{2} \times \nu^{\prime}{ }_{2}\right)^{S} .
$$

We know that $\left(\mu^{\prime} \times \nu^{\prime}\right)^{\mathrm{S}} \leq \rho$ by Theorem $1.1(1)$, so that $(\mu \times \nu)^{\mathrm{S}} \mathrm{Q}\left(\mu^{\prime} \times \nu^{\prime}\right)^{\mathrm{S}}$ [9, Theorem 2.1 (1)]

Our last example shows that the analogue of Theorem 5.4 fails for largest products.

EXAMPLE 5.5. Let $\kappa_{A}$ and $\kappa_{B}$ be the measures on the Borel sets of $X=[0,1]$ given in Example 5.2. Since $\kappa_{A} S \kappa_{B}$, we see that $\kappa_{A} Q \kappa_{B}$. 
of course, $K_{B} Q \kappa_{B}$. Let us show, however, that $\left(\kappa_{A} \times \kappa_{B}\right)^{L}$ is not quasidominant with respect to $\left(\kappa_{B} \times \kappa_{B}\right)^{L}$. Let $D$ be the diagonal of $x \times x$. If $M$ is a Borel set of $X \times X$ such that $\left(K_{A} \times \kappa_{B}\right)^{L}(D)=\left(\kappa_{A} \times \kappa_{B}\right)^{L}(D \cap M)$, then the projection of $M$ has positive Lebesgue measure. Hence, we may choose $z \in B$ such that $(z, z) \in D \cap M$. Then $\left(\kappa_{B} \times \kappa_{B}\right)^{L}(D \cap M)(\{(z, z)\})=1$, even though $\left(\kappa_{A} \times \kappa_{B}\right)^{L} \quad(\{(z, z)\})=0$. It follows that $\left(\kappa_{A} \times \kappa_{B}\right)^{L}$ is not quasi-dominant with respect to $\left(K_{B} \times K_{B}\right)^{L}$.

\section{REFERENCES}

1. $\mathrm{OH}, \mathrm{H}$. S. A note on singularity of measures, Kyungpook Math. J. 11 (1971), 13-16.

2. HALi 10S, P. R. Measure Theory, Van Nostrand, Princeton, N. J., 1950.

3. LUTHER, N. Y. Unique extension and product measures, Canad. J. Math. 19 (1967), 757-763.

4. BERBERIAN, S.K. Measure and Integration, Macmillan, New York, 1965.

5. ROYDEN, H. L. Real Analysis, Macmillan, New York, 1968.

6. JOHNSON, R. A. Extending a measure from a ring to a sigma-ring, Proc. Amer. Math. Soc. 79 (1980), 431-434.

7. LUTHER, N. Y. Lebesgue decomposition and weakly Borel measures, Duke Math. J. 35 (1968), 601-615.

8. JOHNSON, R. A. On the Lebesgue decomposition theorem, Proc. Amer. Math. Soc. 18 (1967), 628-632.

9. JOHNSON, R. A. Some relationships between measures, Pacific J. Math. 82 (1979), 117-132. 


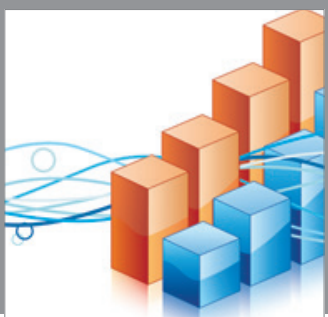

Advances in

Operations Research

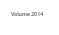

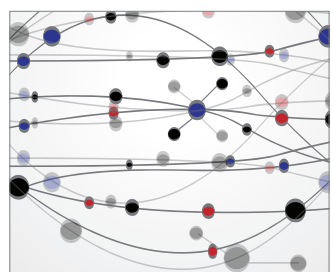

\section{The Scientific} World Journal
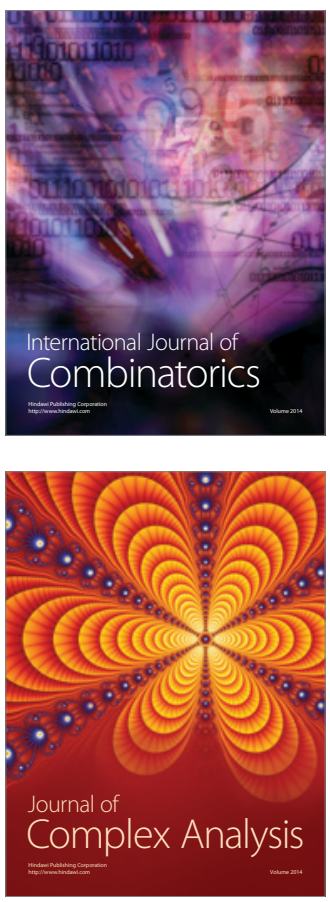

International Journal of

Mathematics and

Mathematical

Sciences
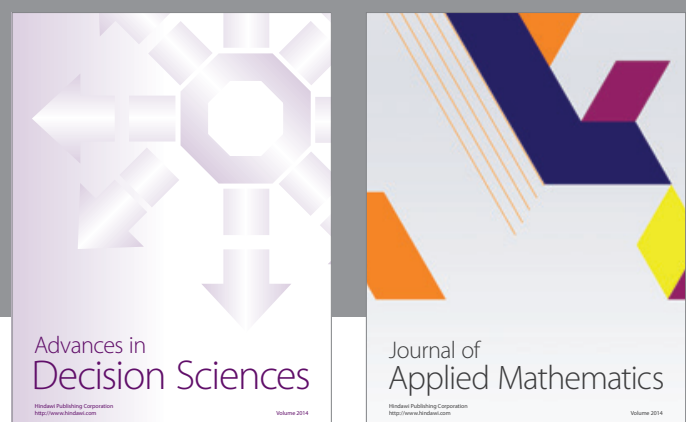

Journal of

Applied Mathematics
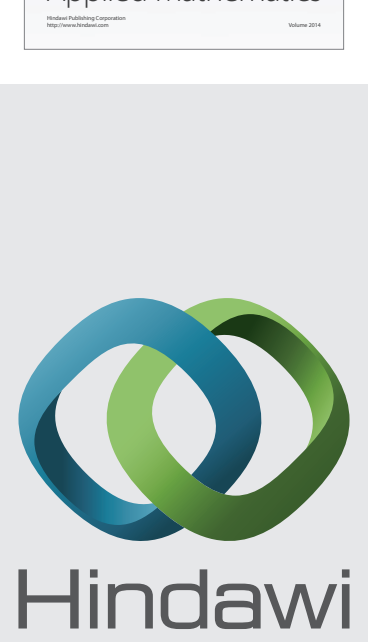

Submit your manuscripts at http://www.hindawi.com
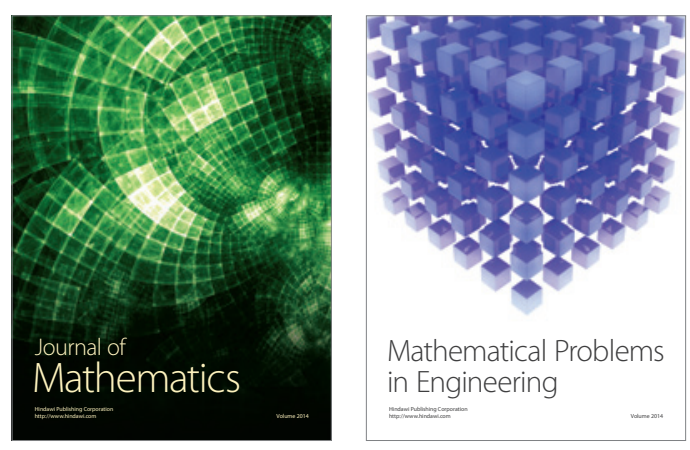

Mathematical Problems in Engineering
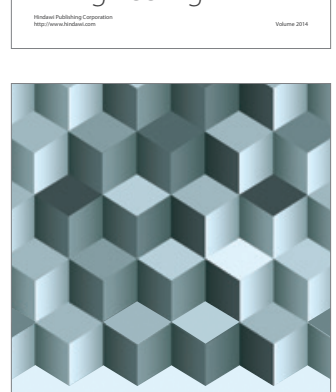

Journal of

Function Spaces
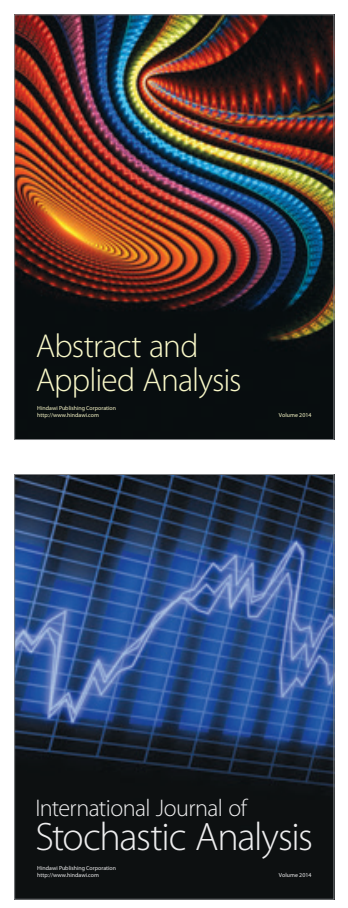

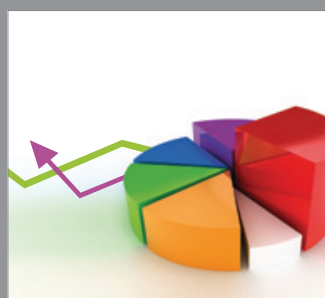

ournal of

Probability and Statistics

Promensencen
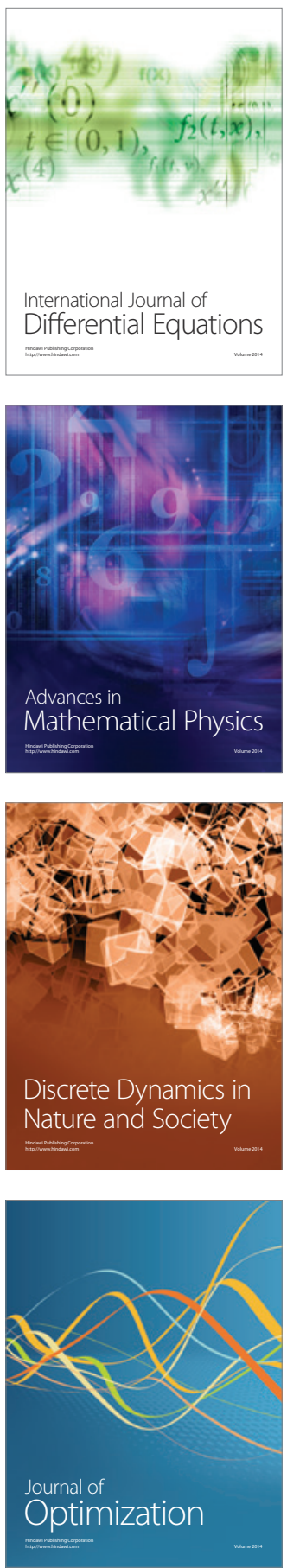